www. revis tad y o. com

\title{
Análisis de la respuesta resiliente de los sectores industriales españoles entre 2008 y 2014
}

The resilient behavior of Spanish industrial sectors (2008-2014): an empirical analysis

\author{
Jesús Manuel Plaza-Llorente \\ Departamento de Economía de la Empresa y Contabilidad. Facultad de Ciencias Económicas y Empresariales. Universidad Nacional de Edu- \\ cación a Distancia (UNED), c/Senda del Rey, 11. 28040 Madrid. \\ jplaza@cee.uned.es
}

Fecha de recepción: 12-11-2014

Fecha de aceptación: 10-04-2015

Resumen: En la medida en que han desarrollado perfiles de resiliencia, los sectores industriales españoles han hecho frente mejor a la crisis económica y financiera 2008-2014. Este hecho, les ha permitido asimilar primero el impacto de la crisis y desarrollar después estrategias adaptativas de respuesta. Los resultados de esta investigación apuntan que, para la orientación de las políticas industrial y de empresa, la experiencia de estos años de crisis resalta la importancia del capital humano, junto con el esfuerzo para mantener operativa la capacidad productiva y comercial de las empresas, como soportes últimos de la resiliencia de los sectores industriales.

Palabras clave: resiliencia, sectores industriales, estrategias adaptativas.

\begin{abstract}
As a consequence of the economic and financial crisis during 2008 and 2014, this paper shows how the Spanish industrial sectors had developed some resilient profiles to tackle the former impact of crisis and react through adaptive strategies. The gain of experience in the period reveals some learning to approach industrial and enterprise policies. The qualification and numbers of human capital, together some efforts to support equipment and marketing operative capacities of firms, are bastions to build Resilience in industrial sectors, as demonstrate an empirical multivariable regression model tested in this article.
\end{abstract}

The measure of Resilience deserves three main approaches into scholar literature. The first, Preventive, reflects how vulnerable is the economic or industrial system, to detail which conditions and intensity of them are possible without any permanent deterioration. Second, Positive, allows a better understanding of behavior of system into a risky or handicapped situations, before it reaches limits of collapse. Finally, a third approach focus on capacities for assessment and evaluation of systems and benchmark, to identify knowledge and learning elements under schemes of Better Practices and Success Cases.

Under a model of lineal multivariable regression had been calculated 12 functions of Resilience (Dependent variable) for other 12 industrial sectors, based on aggregations and data (2008-2014) of National Statistics Office (INE). The robustness of model had been tested at $1 \%$ of confidence level, without significant lineal dependence between independent (regressor) variables (drivers of Resilience), measured through Durbin-Watson's Coefficient values. According results, a $42 \%$ of sectors had a Resilience level upper average (Mean) combined with a low volatility (Standard Deviation). In this group were Chemical \& Pharmaceuticals, Food \& Drinks, Rubber \& Plastics, Motor and Diverse Manufacturing, sectors. The main drivers of this resilient behavior were a stable level of R\&D investment and Purchases into EU. A 16\% of sectors (Extractive Industries and Metals) also shown a resilient profile but volatile (SD upper average), based on Human Capital, a stable effort in R\&D investment and Sales into EU. Finally, other $42 \%$ of sectors (Textile, Machinery, Wood, Electric \& Electronics and No metals industries) were less resilient, based on Human Capital and their capacity to sell into EU.

The results of this work continue a promissory field of research on how to build Resilience, how to increase the role of drivers that provide Resilience into economic and industrial systems and a better understanding on how manage it, nevertheless undesired impacts. The measure of risks and how vulnerability of systems can be afford, based on their own capacities to induce first resilience and recovery after, should be completed with a deeper search of mechanisms to operate into the black box of systems and which tools would be used by industrial and enterprise policies, to sure a faster transit from troubled situations into a recovery and new friendly scenarios for new jobs, confidence and inclusive growth.

Keywords: resilience, industrial sectors, adaptive strategies. 


\section{Introducción}

Desde mediados de 2014, las previsiones de los organismos económicos internacionales y de los responsables de la política económica comienzan a traducirse en cifras indiciarias del fin de ciclo recesivo que, desde 2008 , viene padeciendo la economía española. Llegado este momento, comienzan a aparecer distintos análisis sobre cómo nuestra economía ha transitado por este período y los efectos del impacto que la crisis de la Eurozona, iniciada en 2008, ha tenido sobre ella. En este contexto, el presente artículo contrasta, desde el concepto de Resiliencia, el comportamiento que, entre 2008 y 2014, han tenido los sectores industriales españoles con respecto al papel jugado por la estructura de capital humano y apertura exterior empresariales, señalados ambos como parámetros determinantes del comportamiento de la industria en dicho período.

La Resiliencia es un concepto acuñado por la Física para referirse a la capacidad de recuperación de las propiedades elásticas de un material después de estar sometido a un esfuerzo intenso y prolongado. Como se verá después, al revisar la actualidad de este concepto, la Resiliencia también forma parte hoy del corpus teórico de las Ciencias sociales y, de forma particular del de la Economía, a la hora de explicar el comportamiento de los sectores económicos e industriales frente al ciclo y su respuesta a impactos desestabilizadores, como ha sido la crisis financiera acaecida entre 2008 y 2014. La hipótesis de trabajo que se establece en este artículo para su contraste es que, en la medida en que han inducido perfiles resilientes, los sectores industriales españoles se han enfrentado mejor a la crisis económica y financiera entre 2008 y 2014, lo que les ha permitido asimilar primero el impacto perturbador de la crisis y responder después mediante estrategias adaptativas. Establecida la tesis del artículo y tras este primer apartado introductorio, la segunda sección de este trabajo se dedica a revisar el origen y la actualidad del concepto económico de Resiliencia para, en el tercer apartado, investigar su caracterización mediante variables y modelos especificados a partir de fuentes acreditadas en la literatura. En la cuarta sección del artículo, y mediante la consideración de datos estadísticos procedentes de fuentes de general aceptación, se contrastará la hipótesis de trabajo establecida para los sectores industriales españoles mediante un modelo econométrico, detallando después los resultados obtenidos. Como conclusión, en el quinto apartado de este trabajo, se valorará la contribución de dichos re- sultados a configurar las estrategias seguidas por los sectores industriales en el período objeto de investigación, antes de consignar las referencias bibliográficas consultadas.

\section{Origen y caracterización actual de la Resiliencia económica y empresarial}

El concepto de Resiliencia es, inicialmente, un concepto físico procedente de la Ciencia de los materiales, que se refiere a la propiedad de que gozan algunos de ellos de recuperar su capacidad elástica después de haber sido sometidos a un esfuerzo de fatiga prolongada ${ }^{1}$ (Timoshenko, 1976). En nuestros días, la Resiliencia ha rebasado su ámbito original de aplicación, para referirse tanto a sistemas como a las personas, siendo compartido por las Ciencias Físicas, de la Salud, Biológicas y Sociales. Si, en el caso de los materiales, la investigación de la Resiliencia se focaliza en las estructuras o propiedades endógenas que la hacen posible, cuando su estudio se extiende a los ámbitos de la Medicina, la Ecología o los Sistemas sociales, políticos o económicos, la propia complejidad de los sujetos de investigación ha encontrado un colaborador eficaz en el Enfoque de sistemas (Bertalanffy, 1950) haciendo de éste, el Sistema, la unidad resiliente.

Al revisar el concepto de Resiliencia aplicado a los sistemas microeconómicos, enseguida nos damos cuenta de la importancia que se concede en ellos a las condiciones de entorno o de contexto, a la hora de ponderar la idea de Adaptabilidad, común a todos ellos. En el proceso de ganancia o construcción de resiliencia, los sistemas, a partir de sus características endógenas moduladas por el entorno, adquieren competencia para tratar la adversidad con éxito, superando o recuperándose de sus efectos e impactos negativos (Walsh, 1998). Por su influencia sobre el comportamiento de los sistemas industriales y su gobernanza, constituyen antecedentes de la inducción de Resiliencia la que opera en los ámbitos de la Psicología individual y social y en los Sistemas ambientales, ninguno de ellos ajeno a la realidad empresarial ${ }^{2}$. Más recientemente Sanchis y Poler (2014) han categorizado la naturaleza de las disrupciones inductoras de la resiliencia empresarial, ofreciendo un marco analítico basado en el origen de las disrupciones, los riesgos que las acompañan y sus consecuencias.

\footnotetext{
1"Se denomina Módulo de resiliencia a la cantidad de energía de deformación que por unidad de volumen puede almacenar una barra sin deformación permanente. Si la deformación no alcanza el límite elástico, el trabajo realizado por la fuerza exterior se transforma por completo en energía potencial y puede recobrarse realizando una descarga gradual de la barra deformada". Vid. Timoshenko (1976, $n^{\circ}$ 63, pp. 274-278). ${ }^{2}$ El autor de este trabajo agradece a los evaluadores los comentarios realizados acerca del uso de los términos "empresarial" e "industrial". En términos generales usamos la voz "empresarial" para referirnos a las organizaciones que realizan las actividades "industriales" en cuanto que actividades económicas de transformación. Para los fines de este artículo la voz "empresarial", al ser más amplia, permite resaltar los aspectos organizativos vinculados al Capital humano, relevantes en las conclusiones alcanzadas, como se verá después.
} 
a. Ámbito de la Psicología individual y social

En el ámbito de la Psicología y de la Psicología social el estudio de la Resiliencia busca responder a la pregunta de: ¿por qué algunas personas, grupos o comunidades reaccionan o se adaptan mejor que otros a la adversidad? En este sentido, Robles (2013) define la Resiliencia como la capacidad de los individuos, organizaciones o comunidades expuestos a crisis y vulnerabilidades a anticipar y reducir su impacto, afrontarlo y recuperarse de sus efectos, sin comprometer sus expectativas a largo plazo. La Resiliencia aparece, por tanto, como un constructo multidimensional en el que se suman factores personales, familiares, sociales y contextuales. A partir de la conjunción de estos factores, Jiménez, Izal y Montorio (2014) han configurado un Modelo de Resiliencia aplicable a comunidades productivas bajo la forma de Empresa, integrado por factores internos: convicción de que es posible influir en los resultados de los acontecimientos (Atkinson, Martin y Rankin, 2009), autoestima (Bonanno, 2004), expectativa de que las cosas irán mejor a pesar de las dificultades (Rutter, 2012) y aceptación de la adversidad como conquista moral, y externos, como la presencia de redes sociales o comunitarias en las que el individuo encuentra apoyo material y emocional.

\section{b. Ámbito de los Sistemas ambientales}

Desde los Sistemas ambientales la Resiliencia se presenta con un doble significado. El primero de ellos, es el de la capacidad del sistema para resistir impactos sin que tengan consecuencias a largo plazo (Folke, 2006; Fellmann, 2012; FSIN, 2014) y, el segundo, su capacidad para asimilar impactos sin que haya cambios en la función biológica que desempeña (Walker, Holling, Carpenter y Kinzing, 2004). En el marco del análisis de las consecuencias derivadas del Cambio climático, la $\mathrm{FAO}^{3}$ y la $\mathrm{OCDE}^{4}$ integran la Resiliencia en un marco conceptual más amplio; del que también forman parte el Riesgo, la Vulnerabilidad y la Capacidad adaptativa de los sistemas (FAO-OCDE, 2012). Por extensión y convenientemente modificado dicho marco conceptual, que se esquematiza en la Figura 1, puede aplicarse al conjunto del Sistema económico y a los diferentes subsectores que lo integran (primario, industrial y de servicios).
RIESGOS. Son los impactos reales o potenciales que pueden producir efectos adversos sobre los sistemas económicos y sociales.

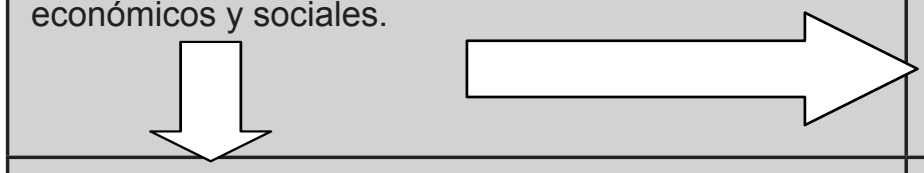

FACULTADES ADAPTATIVAS. Aluden a las posibilidades que residen en cada sistema para hacerse menos vulnerable o soportar mejor el deterioro que pueda sufrir, como consecuencia de impactos bruscos o sostenidos en el tiempo.
VULNERABILIDAD. Concepto dinámico que designa la propensión o predisposición del sistema para recibir impactos adversos, de forma que puedan condicionar o no su estado o desarrollo futuro (Hoddinott y Quisumbing, 2010).

RESILIENCIA. Es la capacidad inducida por el sistema o sus componentes para anticipar, absorber o acomodarse a los efectos de un impacto negativo, recuperando su funcionalidad, asegurando su preservación a largo plazo y restaurando (e incluso mejorando) sus estructuras y funciones propias características.

Figura 1. Marco conceptual explicativo de la Resiliencia de un Sistema económico

\footnotetext{
${ }^{3}$ Agencia de las Naciones Unidas para la Agricultura y la Alimentación (FAO).

${ }^{4}$ Organización para la Cooperación y el Desarrollo Económico (OCDE).
} 
C.

La Resiliencia de los Sistemas económicos

La Resiliencia económica se define como: "la capacidad para mantener los resultados de un Sistema económico, cercanos a su potencial, no obstante las consecuencias de los impactos externos sufridos por el sistema" (Duval, Elmeskov y Vogel, 2007). Por tanto, la Resiliencia económica comprende dos dimensiones fundamentales. La primera se refiere al alcance de los impactos y, la segunda, a la velocidad con que la economía revierte los efectos ocasionados por ellos. Consecuentemente, cabe relacionar la solidez de las políticas estructurales con la capacidad del sistema para soportar los impactos externos y sus efectos, mientras que las políticas de estabilización, están llamadas a jugar un papel determinante en la capacidad de recuperación del sistema (Ekkehard, Gong y Semmer, 2007), como condición necesaria para conseguir el crecimiento económico y nivel de empleo deseados (Aiginger, 2009). Ambos niveles de operación de las políticas -soporte de los impactos y de estabilizacióntambién operan ante la presencia de riesgos globales (WEF, 2015).
De forma particular, la potenciación de las capacidades en los sectores industriales manufactureros, se relacionan con la Innovación, la Eficiencia y el Capital humano (Martín y Segarra, 2005). En este sentido, el Conocimiento y su gestión constituyen claves de diferenciación para la empresa y de su posicionamiento en el mercado (Hidalgo y Castro, 2002; Forés y Camisón, 2008), así como la retención y atracción de conocimiento científico y saber-hacer empresarial (Aceituno, Cea-Moure y Casado, 2013), pasando a constituir actividades críticas en tiempos de crisis económica. Como se representa en la Figura 2, el aumento de la resiliencia en el sistema económico y sus instituciones comporta, además de contar con un modelo de crecimiento inclusivo que aborde el problema del desempleo y la cualificación de la fuerza de trabajo, disponer de mecanismos que disminuyan su vulnerabilidad y potencien la Confianza como intangible favorecedor de su funcionamiento y gobernanza (OCDE, 2014).

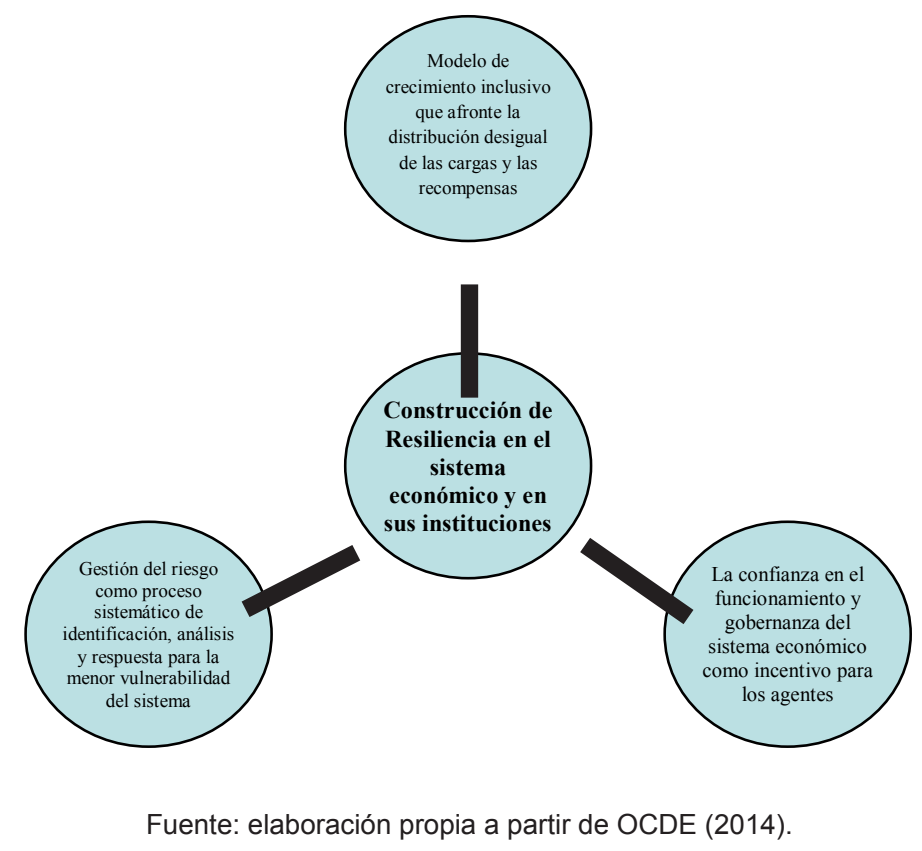

Figura 2. Construcción de Resiliencia en el Sistema económico 


\section{Medida de la Resiliencia económica y empresarial}

La medida de la resiliencia de un sistema económico o industrial obedece a tres enfoques principales recogidos en la literatura. El primero de ellos es de naturaleza Preventiva y, con arreglo a él, se busca definir y cuantificar los elementos de vulnerabilidad que tiene el sistema para, a partir de su conocimiento, determinar en qué condiciones y con qué intensidad podría recibir y estaría en condiciones de afrontar impactos o situaciones desfavorables. El segundo enfoque es de naturaleza Positiva y permite, una vez establecidos los parámetros de resiliencia del sistema y sus límites, valorar su comportamiento en una situación sobrevenida de riesgo o impacto, así como su capacidad de agotamiento $\mathrm{o}$, dicho en otras palabras, determinar el margen de asimilación o de resistencia del sistema a nuevos impactos antes de colapsar. Finalmente, el tercer enfoque es de naturaleza Evaluativa y permite comparar y medir los elementos determinantes de la resiliencia en varios sistemas (benchmarking), valorar su comportamiento relativo y, a partir de ambos, determinar la existencia de elementos de aprendizaje de unos sistemas a partir de otros, mediante la identificación de Mejores prácticas y Casos de éxito. Desde el punto de vista instrumental, la medida de la resiliencia utiliza Métodos semicuantitativos para construir Indices de Resiliencia y Modelos econométricos para la investigación causal de la resiliencia y ponderar (medida de los pesos) las variables o regresores que la determinan. Algunos de los modelos de caracterización y medida de la resiliencia económica mencionados en la literatura, se resumen en la Tabla 1.

Tabla 1. Modelos y medida de la Resiliencia económica y empresarial

\begin{tabular}{|c|c|}
\hline Autores, naturaleza y ámbito del modelo & Variables básicas \\
\hline $\begin{array}{l}\text { Westall, A., Ramsden, P., Foley, J. (2000). } \\
\text { El modelo sintetiza el conjunto de factores que caracterizan el Efecto } \\
\text { multiplicador local como indicador de Resiliencia. } \\
\text { Ámbito de aplicación del modelo: Creación de empresas y su impacto } \\
\text { sobre el desarrollo territorial. }\end{array}$ & $\begin{array}{l}\text { Variable endógena: Efecto multiplicador local. } \\
\text { Variables independientes: } \\
\text { 1. Volumen de bienes y servicios intercambiados } \\
\text { 2. Productores de servicios locales. } \\
\text { 3. Importe de los salarios e inversiones locales. } \\
\text { 4. Construcción de capital social: redes y actividades comunitarias. } \\
\text { 5. Aumento de saber-hacer local. } \\
\text { 6. Apoyos locales al emprendimiento y al empleo. } \\
\text { 7. Promoción y apoyo a la innovación en las empresas y en los } \\
\text { 8. Servicios públicos. }\end{array}$ \\
\hline $\begin{array}{l}\text { Alinovi, L. Mane, E., Romano, D. (2010). } \\
\text { El modelo permite construir un Índice de Resiliencia. Cada variable } \\
\text { básica se determina, a su vez, mediante un número variable de indi- } \\
\text { cadores que la configuran. } \\
\text { Ámbito de aplicación del modelo: Seguridad alimentaria en hogares. }\end{array}$ & $\begin{array}{l}\text { Variable endógena: Índice de Resiliencia. } \\
\text { Variables independientes: } \\
\text { 1. Renta familiar y disponibilidad de alimentos. } \\
\text { 2. Activos productivos: tierra y ganado. } \\
\text { 3. Existencia de redes sociales de seguridad. } \\
\text { 4. Acceso a los servicios básicos: agua potable, electricidad y salud. } \\
\text { 5. Factores adaptativos: educación y posibilidades de diversificación } \\
\text { 6. Estabilidad temporal de las variables anteriores }\end{array}$ \\
\hline Comisión Europea (2012) & $\begin{array}{l}\text { Evaluación del papel jugado por factores institucionales y reformas } \\
\text { en los mercados de productos en el ajuste de la capacidad de los } \\
\text { sectores y de los países a las perturbaciones económicas }\end{array}$ \\
\hline FSIN (2014) & $\begin{array}{l}\text { Variable endógena: Índice normativo de Resiliencia. } \\
\text { Variables independientes: }\end{array}$ \\
\hline $\begin{array}{l}\text { La medida de la resiliencia se fundamenta en la construcción de un } \\
\text { Índice Normativo de Capacidad, construido con arreglo a nueve var- } \\
\text { iables. }\end{array}$ & $\begin{array}{l}\text { 1. Consideración de las percepciones subjetivas. } \\
\text { 2. Enfoque de sistemas y de causalidad compleja. } \\
\text { 3. Vinculación a impactos concretos o específicos } \\
\text { 4. Dependencia de las condiciones iniciales. }\end{array}$ \\
\hline $\begin{array}{l}\text { Por su estructura, se trata de un modelo generalista aplicable a múl- } \\
\text { tiples ámbitos. }\end{array}$ & $\begin{array}{l}\text { 5. Consideración de la volatilidad y comportamiento de los sistemas. } \\
\text { 6. Posibilidad de respuesta fractal, multiescala y multinivel de los } \\
\text { sistemas. } \\
\text { 7. Conexión entre resiliencia y vulnerabilidad. }\end{array}$ \\
\hline
\end{tabular}




\section{Definición, especificación y contraste de un Modelo de Resiliencia para los sectores industriales en España (2008- 2014)}

Para los fines de esta investigación, se elige como sistema económico de referencia el que configuran los Sectores industriales ${ }^{5}$ en España (CNAE 2009), durante el período 2008-2014, siendo el elemento de impacto que ha inducido Resiliencia en cada uno de ellos la crisis financiera iniciada el 14 de septiembre de 2008 , con la quiebra de Lehman Brothers ${ }^{6}$. La etiología y magnitud de esta crisis sobre la industria y la economía en general han sido investigadas por Stiglitz (2011), a la vez que Krugman (2012) ha señalado posibles vías para la recuperación económica general. Por su parte, la lógica del comportamiento de los mercados financieros durante este período, que ha condicionado la respuesta de la industria, ha respondido a los elementos estructurales y operativos analizados por Lacalle (2013). A la luz de los trabajos anteriores cabe señalar que, la respuesta resiliente de los sectores industriales españoles a la crisis en la Eurozona, ha debido producirse fundamentalmente en dos terrenos. En el lado de la Oferta, las empresas han debido afrontar situaciones de racionamiento del crédito y de selección desfavorable como consecuencia de la falta de liquidez y la elevación de la prima de riesgo. Mientras que, en el lado del la Demanda, la caída del consumo y de la cartera de pedidos en el mercado doméstico ha obligado a las empresas a reaccionar con diversas estrategias que, como demostraremos mediante el modelo, han ido desde adaptar la capacidad instalada a las dimensiones del mercado, hasta buscar nuevas oportunidades de negocio y ganar competitividad, manteniendo la actividad productiva y facturando los bienes y servicios comercializados; en definitiva, generando resiliencia.

\footnotetext{
${ }^{5}$ La elección de los Sectores industriales como sistemas objeto de investigación se justifica por dos razones. La primera es de carácter teórico, está acreditada por la literatura y afirma que: cada Sector constituye un agregado sistémico en el que, si bien tienen lugar relaciones de cooperación y competencia entre las empresas integradas en él, por razones estructurales (naturaleza de los aprovisionamientos, tecnologías de producción, naturaleza de los productos y servicios y variables de comercialización, entre otras), muestra un comportamiento diferencial de los restantes sectores; aunque pueda desarrollar circunstancialmente una o varias estrategias comunes. Lo que, dicho en otras palabras, significa que cada Sector industrial puede tener, por razones estructurales, comportamiento y estrategias propias diferenciados de los del resto de sectores. La segunda razón es de carácter instrumental, a partir del hecho de que la fuente estadística usada para contrastar el modelo de Resiliencia propuesto en este artículo (Encuesta Industrial de Empresas del INE), agrupa la información por Sectores industriales.
}

\footnotetext{
6 "En 2008 la economía mundial experimentó cambios trascendentales que quebraron el mayor período de crecimiento continuado desde los años sesenta. Las turbulencias en los mercados financieros internacionales presentes desde el verano de 2007 se transformaron en los últimos meses del año en una profunda crisis financiera global, que ha generando una situación de recesión económica que alcanza ya a todas las principales economías. La escalada de la crisis financiera en septiembre de 2008 intensificó el proceso de desapalancamiento en el que se hallaba inmerso el sistema financiero, al tiempo que agravó sus efectos sobre la actividad real, extendiéndose a las economías emergentes — que hasta ese momento habían dado muestras de cierta resistencia- y provocando que algunos países registraran tasas de crecimiento del PIB muy negativas en el cuarto trimestre del ejercicio pasado y en el primero del actual.” (Banco de España, 2009, pp. 15).
} 


\subsection{Definición y especificación del modelo}

Para identificar la naturaleza de tales estrategias, se propone un Modelo [genérico] de Resiliencia de los Sectores Industriales, integrado por las variables que figuran en la Tabla 2.

Tabla 2. Definición de un Modelo de Resiliencia de los Sectores industriales

\begin{tabular}{|c|c|c|}
\hline \multirow{2}{*}{$\begin{array}{c}\text { Características } \\
\text { de la Resiliencia } \\
\text { como variable } \\
\text { dependiente o } \\
\text { endógena }\end{array}$} & \multicolumn{2}{|c|}{ Regresores (variables independientes) } \\
\hline & Variables teóricas & $\begin{array}{l}\text { Variables operati- } \\
\text { vas o instrumen- } \\
\text { tales }\end{array}$ \\
\hline $\begin{array}{l}\text { Estabilidad. } \\
\text { Supone que } \\
\text { la estructura y } \\
\text { funcionalidad del } \\
\text { sector industrial } \\
\text { permite continuar } \\
\text { su actividad, pese } \\
\text { al impacto. }\end{array}$ & $\begin{array}{l}\text { Capital humano }{ }^{7} \\
\text { sectorial. } \\
\text { Inversión sectorial. } \\
\text { Innovación sec- } \\
\text { torial. }\end{array}$ & $\begin{array}{l}\text { Empleo, personas } \\
\text { ocupadas. } \\
\text { Inversión en activos } \\
\text { materiales. } \\
\text { Inversión en activos } \\
\text { inmateriales. }\end{array}$ \\
\hline $\begin{array}{l}\text { Capacidad adap- } \\
\text { tativa. Se generan } \\
\text { mecanismos de } \\
\text { respuesta del } \\
\text { sistema al impacto } \\
\text { y sus efectos } \\
\text { comunes, siquiera } \\
\text { en parte, para el } \\
\text { conjunto de las } \\
\text { empresas del } \\
\text { sector. }\end{array}$ & $\begin{array}{l}\text { Desarrollo proacti- } \\
\text { vo de capacidades } \\
\text { de supervivencia } \\
\text { y nuevas fuentes } \\
\text { de actividad: } \\
\text { búsqueda de } \\
\text { nuevos mercados } \\
\text { y oportunidades de } \\
\text { negocio. }\end{array}$ & $\begin{array}{l}\text { Ventas en el exte- } \\
\text { rior (UE y resto del } \\
\text { mundo). } \\
\text { Compras en el } \\
\text { exterior (UE y resto } \\
\text { del mundo). }\end{array}$ \\
\hline
\end{tabular}

El contraste del modelo anterior se realizará mediante una regresión lineal multivariante ${ }^{8}$, explicativa de importancia (peso) de cada regresor (valor de los parámetros $\beta$ ) sobre la variable endógena (medidos ambos como tasas interanuales de variación), mediante la especificación detallada en la Tabla 3, con arreglo al modelo econométrico:

$$
y=\beta 0+\sum \beta i x i+u i[1]
$$

Donde y constituye la variable endógena o variable explicada (Resiliencia), xi, los i regresores o variables explicativas, independientes entre sí, extremo éste que se verificará mediante el valor del Coeficiente de Durbin-Watson. Por su parte, el coeficiente $\beta 0$ representa la ordenada en el origen o término independiente de la regresión y ui la perturbación, residuo o error aleatorio. El ajuste se realiza para un Nivel de significación del $1 \%$.

Tabla 3. Especificación de un Modelo lineal de Resiliencia de los Sectores industriales (2008-2014)

\begin{tabular}{|c|c|}
\hline Variable endógena & $\begin{array}{l}\text { Regresores referidos a cada } \\
\text { sector industrial }\end{array}$ \\
\hline $\begin{array}{l}\mathbf{R} \text { (Resiliencia), representa- } \\
\text { da por la Cifra de Negocios } \\
\text { sectorial }(\mathbf{C N}) \text { como variable } \\
\operatorname{proxi}^{9} \text {. }\end{array}$ & $\begin{array}{l}\text { EMP: personas ocupadas. } \\
\text { INVM: inversión en activos } \\
\text { materiales. } \\
\text { INVINT: inversión en activos } \\
\text { intangibles. } \\
\text { V(M): ventas al resto del mun- } \\
\text { do. } \\
\text { V(UE): ventas a la UE. } \\
\text { C(M): compras procedentes } \\
\text { del resto del mundo. } \\
\text { C(UE): compras procedentes } \\
\text { de la UE. }\end{array}$ \\
\hline
\end{tabular}

\footnotetext{
${ }^{7}$ Conjunto de conocimientos, habilidades, destrezas y talentos que poseen las personas ocupadas en la Industria y de las que depende la calidad en el desempeño de su actividad comercial y productiva.

${ }^{8}$ Mediante el software IBM-SPSS Statistics 19, con el método de Entrada de variables.

${ }^{9}$ La Resiliencia, en cuanto tal, no aparece registrada por las fuentes estadísticas como una variable medible. Para los fines de este trabajo se establece la siguiente asociación que justifica el carácter proxy que se le atribuye para el análisis: cuando, no obstante la crisis y sus efectos, las compañías y los sectores industriales siguen facturando es porque, de alguna manera, están asimilando el impacto de la crisis y reaccionando ante ella, es decir, están induciendo Resiliencia. El "cómo" lo está haciendo cada sector, es lo que pretendemos que nos explique el modelo especificado
} 
Los datos, a partir de los cuales se calculan las Tasas interanuales de variación de cada una de las variables ${ }^{10}$, proceden de la Encuesta Industrial de Empresas ${ }^{11}$ del INE (Principales variables económicas por agrupaciones de actividad, en miles de euros, 2012), al nivel de confianza del $1 \%$ en la estimación. La definición de estas variables es la que figura en el apartado 6 (Variables y definiciones) de la metodología de la citada encuesta que, por razones de espacio, no se transcriben aquí.

\subsection{Contraste del modelo y caracter ización de la respuesta resiliente de los sectores industriales españoles (2008-2014)}

\subsubsection{Valores medios y volatilidad de la resiliencia sectorial}

De acuerdo con los valores alcanzados por la Resiliencia media sectorial (R) de la Tabla 4, 7 sectores (el $58 \%$ del total) han tenido un comportamiento superior al del conjunto que, unido a la menor dispersión relativa ${ }^{12}$ de esta variable, hace que los sectores Químico-farmacéutico, de Alimentación y bebidas, Manufacturas diversas, Caucho y plásticos y de Motor y transporte hayan presentado un mejor perfil de resistencia y reacción ante la crisis que ha tenido lugar entre 2008 y 2014. También dos sectores, las Industrias extractivas y la Metalurgia, se han mostrado resilientes, pero con un perfil de riesgo o volatilidad (sigma alta) superior a la media del conjunto de los sectores.
Tabla 4. Resiliencia promedio y volatilidad sectorial Resiliencia media sectorial "R".

\begin{tabular}{|l|r|}
\hline Sector & $\mathbf{R}$ \\
\hline 05-Industrias extractivas & 8,72 \\
\hline 20-Química y farmacia & 1,34 \\
\hline 10-Alimentación y bebidas & 1,02 \\
\hline 31-Manufacturas diversas & $-1,71$ \\
\hline 22-Caucho y plásticos & $-2,22$ \\
\hline $\begin{array}{l}\text { 24-Metalurgia ex-maqui- } \\
\text { naria }\end{array}$ & $-2,93$ \\
\hline 29-Motor y transporte & $-3,10$ \\
\hline Valor medio & $-3,22$ \\
\hline 13-Textil y confección & $-5,61$ \\
\hline 28-Maquinaria & $-7,40$ \\
\hline 16-Madera y corcho & $-7,48$ \\
\hline 26-Material y equipo EEO & $-7,53$ \\
\hline 23-Minerales no metálicos & $-11,78$ \\
\hline
\end{tabular}

\section{Desviación típica de "R".}

\begin{tabular}{|l|r|}
\hline Sector & Sigma \\
\hline 10-Alimentación y bebidas & 5,18 \\
\hline 13-Textil y confección & 7,49 \\
\hline 20-Química y farmacia & 8,41 \\
\hline 29-Motor y transporte & 8,70 \\
\hline 23-Minerales no metálicos & 10,27 \\
\hline 22-Caucho y plásticos & 10,55 \\
\hline 26-Material y equipo EEO & 11,17 \\
\hline 31-Manufacturas diversas & $\mathbf{1 1 , 9 8}$ \\
\hline Valor medio & 12,01 \\
\hline 16-Madera y corcho & 12,88 \\
\hline 28-Maquinaria & 13,31 \\
\hline 05-Industrias extractivas & 19,92 \\
\hline $\begin{array}{l}\text { 24-Metalurgia ex-maqui- } \\
\text { naria }\end{array}$ & 24,24 \\
\hline
\end{tabular}

\footnotetext{
${ }^{10}$ Una serie de tasas de variación está libre de tendencia (Álvarez, 1998; pp. 87), por lo que solo refleja el comportamiento cíclico de las variables

${ }^{11}$ Para disponer de la serie estadística completa desde 2007-8 a 2013-14 se ha recurrido a fuentes complementarias de datos, debido a cambios metodológicos en la encuesta original, o necesarias para su estimación. A tal fin, se ha contado con las siguientes fuentes adicionales: Encuesta sobre innovación tecnológica en las empresas 2007 (INE), Central de Balances del Banco de España 2012 (Cuadro 2.8) y Actualización del Programa de Estabilidad 2014-2017 del Reino de España.

12 La dispersión de la Resiliencia sectorial se mide mediante su Desviación típica (Sigma). A este valor se le asigna un doble significado. En primer lugar, es indicativo de la posible oscilación o volatilidad de la variable a que se refiere (Resiliencia sectorial) y, en segundo lugar, representa el riesgo de que ésta se aparte del valor promedio sectorial (Media aritmética).
} 


\subsubsection{Contribución de las variables explicativas al comportamiento resiliente sectorial (2008-2014)}

Para el nivel del significación del 1\%, el modelo lineal ensayado es robusto para explicar el fenómeno investigado (la forma en que los sectores industriales han inducido Resiliencia a través del comportamiento de sus regresores) con un buen ajuste de éstos y niveles aceptables de multicolinealidad entre ellos, al mantenerse los coeficentes de Durwin-Watson (DW) entre 1,5 y 2,5 para la mayor parte de los sectores (Tabla 5). A partir del valor de los Coeficientes Beta estandarizados $(\beta)$ de los regresores, y para el conjunto de los sectores industriales, las Ventas realizadas fuera de la UE $(0,50)$, las Inversiones materiales $(0,48)$, el Capital humano-empleo $(0,37)$ y las Compras comunitarias $(0,19)$, son las variables que han contribuido positivamente a generar resiliencia sectorial entre 2008 y 2014, mientras que la menor innovación e inversiones en activos intangibles $(-0,34)$, las ventas a la UE $(-0,21)$ y las compras al resto del mundo $(-0,15)$, han detraído capacidad resiliente sectorial en el periodo, en términos agregados. Con todo, el saldo neto de la contribución media a la resiliencia sectorial de las variables implicadas en la internacionalización de la producción y comercialización empresariales a la UE y al resto del mundo, ha resultado positiva ${ }^{13}$ (beta de 0,33 ).

Por variables explicativas, las Compras realizadas fuera de la UE, es la variable que más veces ha resultado excluida del modelo, siendo asimismo baja su contribución a la resiliencia media para el conjunto de los sectores, medida por el valor de su coeficiente beta estandarizado $(-0,15)$. Sin embargo, cuando el análisis se realiza sector a sector, el peso de esta variable es determinante en sectores fuertemente dependientes del exterior para su abastecimiento de materias primas, como sucede con las Industrias extractivas (beta de 1,60$)$. En cuanto a la importancia relativa de la ponderación de los regresores en los cinco sectores industriales para los que la resiliencia entre 2008 y 2014 ha sido mayor y más estable (Químico-farmacéutico, Alimentación y bebidas, Manufacturas diversas, Caucho y plásticos y de Motor y transporte), los resultados muestran comportamientos diferenciales, cuyo alcance último -de naturaleza estructural-, deberá ser abordado con mayor precisión por futuras investigaciones. Con carácter preliminar, el modelo muestra que en el sector Químico-farmaceútico la resiliencia se ha debido mayoritariamente al capital humano, las compras a la UE y las ventas al resto del mundo. En el caso de la Industria alimentaria, han sido las inversiones (materiales e inmateriales), el capital humano y la internacionalización sectorial las fuentes de resiliencia. Otros comportamientos han sido los de las Manufacturas diversas, cuya resilencia se ha debido principalmente a las inversiones materiales e imateriales y a la compra de materias primas en el exterior, y los de los dos sectores restantes, en los que la resiliencia se ha debido con preferencia a las compras foráneas de materias primas (Caucho y plásticos) y a su internacionalización (Motor y transporte).

Dichos comportamientos diferenciales entre los sectores, se aprecian de manera más nítida cuando el análisis se realiza teniendo en cuenta sólo las situaciones en las que la contribución de cada variable explicativa a la Resiliencia es igual o superior a la media de cada sector, como recoge la Tabla 6.

\footnotetext{
${ }^{13}$ Valor $\beta$ neto de ventas y compras en el exterior (Resto del mundo y UE) para el conjunto de los sectores industriales $=0,50-0,21-0,15$
} $+0,19=0,33$. 
Tabla 5. Modelo de ajuste lineal para un nivel de significación del 1\%.

Coeficientes Beta estandarizados de las variables independientes explicativas o regresores.

\begin{tabular}{|c|c|c|c|c|c|c|c|c|}
\hline $\begin{array}{l}\text { Sector/Coeficientes } \\
\text { Beta }\end{array}$ & Empleo & $\begin{array}{l}\text { I. Materi- } \\
\text { ales }\end{array}$ & $\begin{array}{l}\text { I. Intangi- } \\
\text { bles }\end{array}$ & $\begin{array}{l}\text { Ventas } \\
\text { (M) }\end{array}$ & $\begin{array}{l}\text { Ventas } \\
\text { (UE) }\end{array}$ & $\begin{array}{l}\text { Compras } \\
\text { (M) }\end{array}$ & $\begin{array}{l}\text { Compras } \\
\text { (UE) }\end{array}$ & $D W(2)$ \\
\hline $\begin{array}{l}\text { 05-Industrias extrac- } \\
\text { tivas }\end{array}$ & $-0,28$ & 0,25 & $-0,26$ & Ex (1) & $-2,60$ & 1,60 & 2,29 & 1,79 \\
\hline $\begin{array}{l}\text { 10-Alimentación y } \\
\text { bebidas }\end{array}$ & 0,26 & 1,12 & 0,27 & 0,51 & 0,02 & Ex (1) & 0,05 & 2,46 \\
\hline 13-Textil y confección & 0,90 & 1,77 & Ex (1) & 0,62 & $-0,77$ & $-0,46$ & $-0,96$ & 2,35 \\
\hline 16-Madera y corcho & 0,19 & 1,99 & $-0,95$ & Ex (1) & 1,05 & $-0,98$ & $-1,08$ & 2,32 \\
\hline $\begin{array}{l}\text { 20-Química y farma- } \\
\text { cia }\end{array}$ & 0,51 & 0,24 & $-0,81$ & 0,64 & $-0,43$ & Ex (1) & 0,89 & 2,70 \\
\hline $\begin{array}{l}\text { 22-Caucho y plásti- } \\
\text { cos }\end{array}$ & 0,29 & $-1,04$ & $-0,40$ & $-0,06$ & Ex (1) & $-0,84$ & 2,16 & 2,64 \\
\hline $\begin{array}{l}\text { 23-Minerales no } \\
\text { metálicos }\end{array}$ & 0,77 & 0,05 & $-0,55$ & 1,15 & 0,44 & Ex (1) & $-0,71$ & 2,59 \\
\hline $\begin{array}{l}\text { 24-Metalurgia } \\
\text { ex-maquinaria }\end{array}$ & 0,38 & Ex (1) & $-0,14$ & 1,40 & 0,15 & 0,06 & $-0,98$ & 2,89 \\
\hline $\begin{array}{l}\text { 26-Material y equipo } \\
\text { EEO }\end{array}$ & 0,54 & $-0,07$ & $-0,25$ & 0,43 & Ex (1) & $-0,22$ & $-0,86$ & 1,78 \\
\hline 28-Maquinaria & 0,69 & 0,13 & $-0,48$ & 0,48 & 0,35 & Ex (1) & $-0,07$ & 1,44 \\
\hline 29-Motor y transporte & 0,26 & $-0,20$ & $-0,16$ & 0,56 & $-0,12$ & Ex (1) & 0,70 & 2,37 \\
\hline $\begin{array}{l}\text { 31-Manufacturas } \\
\text { diversas }\end{array}$ & $-0,11$ & 0,99 & $-0,04$ & $-0,72$ & Ex (1) & $-0,21$ & 0,82 & 0,97 \\
\hline $\begin{array}{l}\text { Valor medio Betas } \\
\text { y DW }\end{array}$ & 0,37 & 0,48 & $-0,34$ & 0,50 & $-0,21$ & $-0,15$ & 0,19 & 2,19 \\
\hline
\end{tabular}

(1) Ex: variable excluida por el ajuste, (2) Coeficiente de Durwin-Watson.

Fuente: elaboración propia, a partir de los resultados de cada una de las 12 funciones de regresión obtenidas. Las salidas de software, así como los datos que están en el origen de los coeficientes mostrados en este cuadro, se ponen por el investigador a disposición de quien lo solicite. 
De esta manera, aparecen cuatro zonas de "concentración de resiliencia" asociadas al nivel de resiliencia y su estabilidad (valores de la desviación típica). Bajo la categoría de Resilientes estables (42\%) se han agrupado los sectores de elevada resiliencia relativa y baja volatilidad (sigma pequeña), como Resilientes Volátiles (16\%) se agrupan los sectores de resiliencia y desviación típica altas. Finalmente, y como tercera categoría, se han agrupado los sectores de menor resiliencia relativa $(42 \%)$.

Tabla 6. Modelo de ajuste lineal (1\%).

Valores de los coeficientes Beta estandarizados. "R" indica: "resiliencia igual o superior a la media de Beta para cada variable y sector".

\begin{tabular}{|c|c|c|c|c|c|c|c|}
\hline Clasificación sectorial & Empleo & I. Mat. & I. Intang. & $\mathrm{V}(\mathrm{M})$ & $\mathrm{V}(\mathrm{UE})$ & $\mathrm{C}(\mathrm{M})$ & $\mathrm{C}(\mathrm{UE})$ \\
\hline \multicolumn{8}{|l|}{ RESILIENTES ESTABLES } \\
\hline 20-Química y farmacia & $\mathrm{R}$ & & & $\mathrm{R}$ & & & $\mathrm{R}$ \\
\hline 10-Alimentación y bebidas & & $\mathrm{R}$ & $\mathrm{R}$ & & $\mathrm{R}$ & & \\
\hline 31-Manufacturas diversas & & $\mathrm{R}$ & $\mathrm{R}$ & & & & $\mathrm{R}$ \\
\hline 22-Caucho y plásticos & & & & & & & $\mathrm{R}$ \\
\hline 29-Motor y transporte & & & $\mathrm{R}$ & $\mathrm{R}$ & $\mathrm{R}$ & & $\mathrm{R}$ \\
\hline \multicolumn{8}{|l|}{ RESILIENTES VOLÁTILES } \\
\hline 05-Industrias extractivas & & & $\mathrm{R}$ & & & $\mathrm{R}$ & $\mathrm{R}$ \\
\hline 24-Metalurgia ex-maquinaria & $\mathrm{R}$ & & $\mathrm{R}$ & $\mathrm{R}$ & $\mathrm{R}$ & $\mathrm{R}$ & \\
\hline \multicolumn{8}{|l|}{ MENOS RESILIENTES } \\
\hline 13-Textil y confección & $\mathrm{R}$ & $\mathrm{R}$ & & $\mathrm{R}$ & & & \\
\hline 28-Maquinaria & $\mathrm{R}$ & & & & $\mathrm{R}$ & & \\
\hline 16-Madera y corcho & & $\mathrm{R}$ & & & $\mathrm{R}$ & & \\
\hline 26-Material y equipo EEO & $\mathrm{R}$ & & $\mathrm{R}$ & & & & \\
\hline 23-Minerales no metálicos & $\mathrm{R}$ & & & $\mathrm{R}$ & $\mathrm{R}$ & & \\
\hline
\end{tabular}


De acuerdo con la clasificación y análisis de la Tabla 6 , el Capital humano (empleo) es determinante para conseguir resiliencia en sectores desfavorecidos por la presencia de Inversiones materiales e Intangibles, por lo que estaríamos ante un trade-off clásico entre los factores de Capital y Trabajo. Por su parte, la innovación y la inversión en intangibles empresariales (gastos en I+D, patentes y modelos de utilidad, marcas y reputación comercial, concesiones, aplicaciones informáticas y otros) aparece vinculada a los sectores industriales de mayor resiliencia relativa. Finalmente, la internacionalización sectorial es fuente determinante de resiliencia; si bien, en el caso de los sectores más resilientes, contribuye más a través de la ganancia de competitividad en la gestión de las compras y aprovisionamientos procedentes de la UE mientras que, en los sectores de menor resiliencia relativa, lo hace a través de la ganancia de competitividad en las ventas de productos y servicios a esos mismos mercados comunitarios.

\section{Conclusiones}

La aplicación del concepto de Resiliencia, poco frecuente para la investigación causal del comportamiento de los sectores industriales, realizada en este artículo para el caso español entre 2008 y 2014, se ha mostrado válida para modelizar y explicar su comportamiento en respuesta a la crisis acaecida en dicho período. El impacto causado por la Crisis de la Eurozona y sus consecuencias durante el período investigado, inicialmente ha inducido mecanismos de supervivencia en los sectores industriales que, con posterioridad, han desarrollado estrategias adaptativas de medio plazo. De acuerdo con el modelo multivariante contrastado, y como respuesta inicial a la crisis, los sectores industriales han implementado una estrategia genérica que, con diferentes grados de intensidad, ha supuesto el ajuste estructural en torno a las variables de Capital humano e Inversiones en intangibles. En términos comparativos, el empleo industrial sectorial ha contribuido de forma positiva a la resiliencia sectorial, excepto en el caso de las Industrias extractivas y las Manufacturas diversas, en las que su resiliencia se ha debido, comparativamente más, a la ganancia de competitividad a través de la gestión de los aprovisionamientos procedentes de la Unión Europea. Por lo que se refiere a las Inversiones en intangibles, su contribución a la resi- liencia sectorial global de los 12 sectores investigados durante 2008 y 2014 ha sido negativa, aunque sectores como el Alimentario, las Manufacturas diversas, el Metalúrgico y el de Motor y transporte han mantenido niveles de inversión superiores a los del resto de los sectores, en términos comparativos.

Una vez asimilado el impacto de la crisis, y en sintonía con la recesión en el mercado doméstico, entre 2008 y 2014, los sectores industriales se han adaptado a las nuevas condiciones de mercado desarrollando dos estrategias resilientes para mantener la actividad en el medio plazo, que han sido explicadas por el modelo contrastado en este artículo. La primera de ellas ha sido la de atenuar, cuanto han podido, la caída de la inversión en utillaje y equipo (Inversiones materiales), seguramente para poder aprovechar las oportunidades que ofrecían los mercados exteriores, en lo que ha sido la segunda estrategia adaptativa llevada a la práctica. Además del Capital humano (Empleo), entre 2008 y 2014, las Inversiones materiales han contribuido de forma definitiva a la resiliencia de los sectores de Madera y corcho, Textil-confección y de Alimentación y bebidas y, en menor grado, a la de los sectores Extractivo y Químico. En términos relativos, los sectores Maderero, de Minerales no metálicos, de Maquinaria y Metalúrgico son los que mejor han podido posicionar sus productos en los mercados exteriores, con ventaja sobre los Extractivo, de Caucho y plásticos, Químico, de Manufacturas diversas y de Motor y transporte, cuya ganancia de resiliencia comparativa ha tenido lugar a través de los aprovisionamientos de materias primas y componentes de procedencia comunitaria. Los resultados de esta investigación apuntan que, para la orientación de las políticas industrial y de empresa, la experiencia de estos años de crisis económica e industrial pone de relieve la importancia del capital humano en cantidad y cualificación suficientes, junto con el esfuerzo para mantener operativa la capacidad productiva y comercial de las empresas, como soportes últimos de la resiliencia de los sectores industriales; en el sentido de que, en ambas variables, reside la capacidad inicial para asimilar impactos perturbadores simultáneos en el lado de la oferta y en el de la demanda, a la vez que constituyen las palancas que, con posterioridad, sirven para aprovechar las oportunidades de mercado inducidas por las ganancias de competitividad y la creación de nuevo valor aportado por la innovación y la I+D. 


\section{Bibliografía}

ACEITUNO, P., CEA-MOURE, R., CASADO, J. (2013): "Políticas de esfuerzo combinado económico-laboral en la retención y atracción del conocimiento científico: un estudio del caso español. Dirección y organización, nº 49, pp. 54-63.

AIGINGER, K., (2009): "Strenthening the resilience of an economy". S:/Applic/WEB/NERO/September 2009/Background information aiginger.docx .

ALINOVI, L. MANE, E., ROMANO, D. (2010): "Meausuring household resilience to food insecurity: an application to Palestinian households". Agricultural Survey Methods. John Wiley \& Sons, April 2010.

ÁLVAREZ, N. (1998): Addenda de Econometría. UNED.

ATKINSON, P., MARTIN, C., RANKIN, J. (2009): "Resilience revisited". Journal of psychiatric and mental health nursing, 16, pp. 137-145.

BANCO DE ESPAÑA (2009): Informe anual 2008.

BERTALANFFY, L. (1950): "An Outline of General System Theory". British Journal for the Philosophy of Science 1, pp. 139-164.

BONANNO, G. (2004): "Loss, trauma and human resilience: have we underestimated the human capacity to thrive extremely aversive events?" American psychology, 59(1), pp. 20-28.

CATTE, P., GIROUARD, N., PRICE, R., ANNDRÉ, $\mathrm{CH}$. (2004): The contribution of housing markets to cyclical resilience. OECD Economic Studies, $\mathrm{n}^{\circ}$ $38,2004 / 1$.

COMISIÓN EUROPEA (2012): Measuring the macroeconomic resilience of industrial sectors in the $E U$ and assessing the role of product market regulations. Occasional papers 112.

COMISIÓN EUROPEA (2014): Investigación e innovación como fuentes de un crecimiento renovado. Comunicación de la Comisión COM(2014) 339 final. Bruselas 10 de junio de 2014.
DUVAL, R., ELMESKOV, J., VOGEL, L. (2007): Structural policies and economic resilience to shocks. OECD Workshop on structural reforms and economic resilience: evidence and policy implications. June 2007.

EKKEHARD, E., GONG, G., SEMMER, W. (2007): Resilience, consumption smoothing and structural policies. OECD Workshop on structural refor$\mathrm{ms}$ and economic resilience: evidence and policy implications. June 2007.

FAO-OCDE (2012): Building resilience for adaptation to climate change in the agriculture sector. Proceedings of a Joint FAO/OECD Workshop 23-24 April 2012.

FELLMANN, T. (2012): The assesment of climate change related vulnerability in the agricultural sector: reviewing conceptual frameworks. FAOOECD workshop: Building resilience for adaptation to climate change in the agricultural sector. Roma, 23-24 April, 2012.

FOLKE, C. (2006): "Resilience: the emergence of a perspective for social-ecological systems analysis". Global environmental change, 16, pp. 253267.

FOOD SECURITY INFORMATION NETWORK (FSIN) (2014): Resilence measurement principles. Technical series no 1. Enero 2014.

FORÉS, J., CAMISÓN, C. (2008): "La capacidad de absorción de conocimiento: factores determinantes internos y externos". Dirección y organización, $n^{\circ} 36$, pp. 35-50.

GRENOILLEAU, D., RATTO, M., ROEGER, W. (2007): Adjustment shocks: a comparison between the Euro area and the US using estimated DSGE models. Comisión Europea, junio 2007.

HIDALGO, A., CASTRO, F. (2002): "Hacia la organización del futuro basada en el conocimiento". Dirección y organización (27), pp. 27-43. 
HODDINOTT J., QUISUMBING A. (2010): Methods for microeconometric risk and vulnerability assessment in Risk, Vulnerability and Human Development: On the brinke, by Fuentes-Nieva R. and Seck P. London: Palgrave Macmillan - United Nations Development Programme (UNDP).

JIMENEZ, M., IZAL, M., MONTORIO, I. (2014): "Psychological and social factors that promote positive adaptation to stress and adversity in adult life cycle". Journal of happiness studies, Vol13, Issue, pp.833-848.

KARFAKIS, P. (2012): The assessment of the socio-economic impacts of climate change at household level. FAO-OCDE Workshop: Building resilience for adaptation to climate change in the agriculture sector. Rome, 23-24 April 2012.

KRUGMAN, P. (2012): ¡Acabad ya con esta crisis! Planeta.

LACALLE, D. (2013): Nosotros los mercados. Deusto.

LEVINA, E., TIRPAK, D. (2006): Adaptation to climate change: key terms. OCDE, 9 de mayo de 2006.

MARTÍN, M., SEGARRA, A. (2005): "Dinámica empresarial en las industrias maduras españolas". Economía Industrial, nº 355-356.

ORGANIZACIÓN PARA LA COOPERACIÓN Y EL DESARROLLO ECONÓMICO (OCDE) (20132014): OECD Year book 2013-2014. París.

ORGANIZACIÓN PARA LA COOPERACIÓN Y EL DESARROLLO ECONÓMICO (OCDE) (2014): Boosting Resilience through Innovative Risk Governance. París.
ROBLES, J. (2013): "Manejo de resiliencia y vulnerabilidad intrínsecos al ser humano". Lex artis ad hoc. International scientific journal, $n^{\circ} 2$.

RUTTER, M. (2012): "Resilience as a dynamic concept". Development and psychopathology, 24, pp. 335-344. Cambridge university press.

SANCHIS, R.; POLER, R. (2014): "Enterprise resilience assessment: a categorization framework of disruptions". Dirección y organización (54), pp. 4553.

STGLITZ, J. (2011): Caída libre. Santillana ediciones generales.

TIMOSHENKO, S. (1976): Resistencia de materiales. Espasa-Calpe.

WALKER, B., HOLLING, C., CARPENTER, S. Y KINZING, A. (2004): "Resilience. Adaptabilility and transformability in social-ecological systems”. Ecology and society 9(2):5.

WALSH, F. (1998): Strengthening family resilience. Guilford press.

WESTALL, A., RAMSDEN, P. Y FOLEY, J. (2000): Micro-entrepreneurs: creating enterprising communities. IPPR and NEF, London.

WORLD ECONOMIC FORUM (WEF) (2015): Global risk 2015. 10th Edition. Insight Report. 